\title{
Using active contour models for feature extraction in camera-based seam tracking of arc welding
}

\author{
Liu, Jinchao; Fan, Zhun; Olsen, Søren; Christensen, Kim; Kristensen, Jens
}

Published in:

International Conference on Intelligent Robots and Systems, 2009. IROS 2009. IEEE/RSJ

Link to article, DOI:

$10.1109 /$ IROS.2009.5354390

Publication date:

2009

Document Version

Publisher's PDF, also known as Version of record

Link back to DTU Orbit

Citation (APA):

Liu, J., Fan, Z., Olsen, S., Christensen, K., \& Kristensen, J. (2009). Using active contour models for feature extraction in camera-based seam tracking of arc welding. In International Conference on Intelligent Robots and Systems, 2009. IROS 2009. IEEE/RSJ IEEE. https://doi.org/10.1109/IROS.2009.5354390

\section{General rights}

Copyright and moral rights for the publications made accessible in the public portal are retained by the authors and/or other copyright owners and it is a condition of accessing publications that users recognise and abide by the legal requirements associated with these rights.

- Users may download and print one copy of any publication from the public portal for the purpose of private study or research.

- You may not further distribute the material or use it for any profit-making activity or commercial gain

- You may freely distribute the URL identifying the publication in the public portal 


\title{
Using Active Contour Models for Feature Extraction in Camera-Based Seam Tracking of Arc Welding
}

\author{
J. Liu, Z. Fan, S. Olsen, K. Christensen and J. Kristensen
}

\begin{abstract}
In the recent decades much research has been performed in order to allow better control of arc welding processes, but the success has been limited, and the vast majority of the industrial structural welding work is therefore still being made manually. Closed-loop and nearly-closed-loop control of the processes requires the extraction of characteristic parameters of the welding groove close to the molten pool, i.e. in an environment dominated by the very intense light emission from the welding arc. The typical industrial solution today is a laser-scanner containing a camera as well as a laser source illuminating the groove by a light curtain and thus allowing details of the groove geometry to be extracted by triangulation. This solution is relatively expensive and must act several centimetres ahead of the molten pool. In addition laser-scanners often show problems when dealing with shiny surfaces. It is highly desirable to extract groove features closer to the arc and thus facilitate for a nearly-closed-loop control situation. On the other hand, for performing seam tracking and nearly-closed-loop control it is not necessary to obtain very detailed information about the molten pool area as long as some inportant features are obtained, e.g. the groove position and gap width. To obtain these features without external illumination, a new image analysis scheme based on active contour models was proposed and verified by experimental results.
\end{abstract}

\section{INTRODUCTION}

For decades, welding processes have been used widely in many important industial applications. However, welding processes produce airborne particles and gaseous by-products which can be harmful to human health. Therefore, welding automation/robotics are highly required in the industrial environment. Due to the complex nonlinear process of arc welding and the lack of sensors which can extract sufficient information of weld process for control, most arc welding robotics are not equipped with a close-loop control system. Due to the limitations of the traditional sensors such as Xray radiation, ultrasonic, etc, used in the welding industry today, non-contact camera-based sensor systems have been attracting more and more researchers[1], [2], [3].

In welding seam tracking, sensors based on structured light play an important role due to their simplicity, applicability to perform in real-time, and immunity to the interference of high intensity arc light. Haug and Pritschow [2] proposed

J. Liu is with Department of Management Engineering, Technical University of Denmark, 2800 lyngby, Denmark. He is also affiliated with FORCE Technology, 2650 Broenby, Denmark. jliu@man.dtu.dk

Z. Fan is with Department of Management Engineering, Technical University of Denmark, 2800 lyngby, Denmark. zhfa@man.dtu.dk

S. Olsen is with Department of Computer Science, University Of Copenhagen, 2100 Copenhagen, Denmark. ingvor diku.dk

K. Christensen is with Division of Welding \& Production Innovation, FORCE Technology, DK-2605 Broenby, Denmark. kmc@force.dk

J. Kristensen is with Division of Welding \& Production Innovation, FORCE Technology, DK-2605 Broenby, Denmark. jek@force.dk a low-cost laser stripe sensor system for the automation of welding processes which used only one highly integrated logic IC to avoid using expensive multiprocessor sensor. They also developed a new robust fuzzy algorithm to detect the location of the welding groove. In [3], Xu et al. proposed a new scheme of image processing to extract geometrical features of the welding seam for structured light images with arc and splash disturbances. As a typical industrial solution, vision sensors based on laser scanner and structured light are also available in commercial market.

Although laser scanner has found its important use in welding seam tracking, according to our own practical experiences, we have observed the following limitations: i) the laser scanner is still relatively expensive, ii) it does not perform well when it is close to the molten pool, iii) it can cause problems when dealing with shiny surfaces. The limitations motivated us to develop a new camera-based solution without external illumination.

The main challenge encountered during vision based monitoring near the molten pool is the strong interference from the high-intensity arc light across a wide spectrum as well as spatters arising from the welding process (illustrated in Fig. 1). As a solution with external laser illumination, laser scanner utilizes the monochromatic property of laser light that filters out the high-intensity interference using narrow band-pass filters. Without external illumination, the key to groove features extraction close to arc is to overcome the interference using only image analysis techniques. It should be noted that in this paper pipe-welding was adopted and the joint was V-Groove, as seen in Fig. 1(a). From the image captured by the camera which is mounted on the top of the VGroove, four edges(lines) of V-Groove should be extracted, as shown in Fig. 1(c). After camera calibrating, we can calculate the groove position and gap width using these four lines. In this paper, a new image analysis scheme based on active contour models for seam tracking is proposed. Experimental results are provided and demonstrate the feasibility of this approach

\section{A Novel Image Analysis Scheme for Seam TRACKING}

A typical technique to extract the lines from images is Hough transform [4], and in many cases an edge detector [6] can also be use to pre-processing the images. From Fig. 1(b), it can be seen that seam images captured by normal speed camera without external illumination during welding are seriously corrupted by high-intensity arc light and spatters. This makes it difficult for traditional edge detection + Hough 
outer line 1 outer line 2

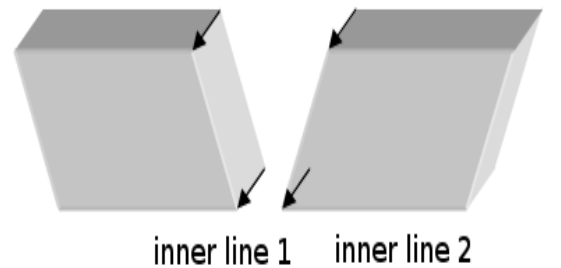

inner line 1 inner line 2

(a)

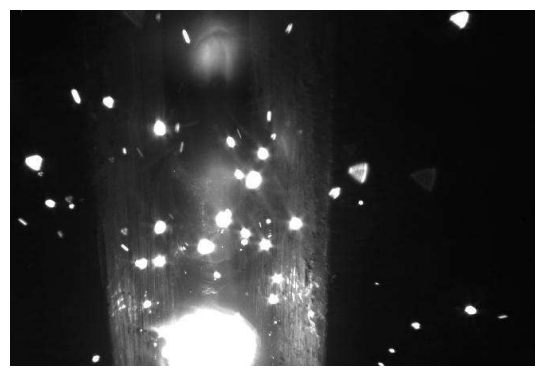

(b)

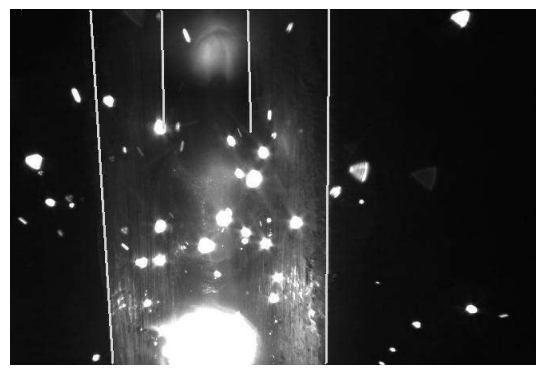

(c)

Fig. 1. (a) Four edges of V-Groove.(b) Image captured during welding, with spatters. (c) The edges of the groove which needs to be extracted marked by white lines manually. The inner two lines represent lower edges of the V-Groove, the outer two lines represent upper edges of the V-Groove. The width of the V-Groove can be computed after camera calibration.

transform technique to find the edges of the groove. Fig. 2 shows the results of applying this technique to the image shown in Fig. 1. In this paper, we propose a new image analysis scheme based on active contour models to extract the edges of the seams.

\section{A. Snakes: Active Contour Models}

Snake was first introducted by Kass et al. in 1988 as active contour model [5]. Since then, it has been widely used in computer vision and image processing in applications including edge detetion, image segmentation and motion tracking, etc. It aims to locate the contour of an object driven by internal, external, and image dependent forces.

The traditional active contour model is represented as a parametric curve $\mathbf{u}(s)=(x(s), y(s))$, where $s \in[0,1]$.

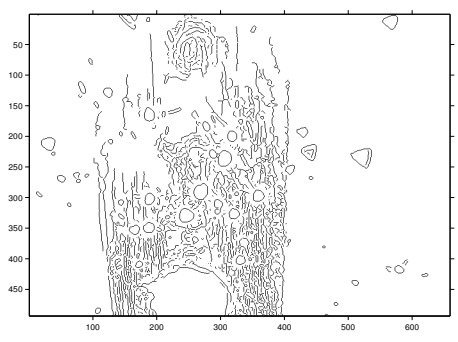

(a)

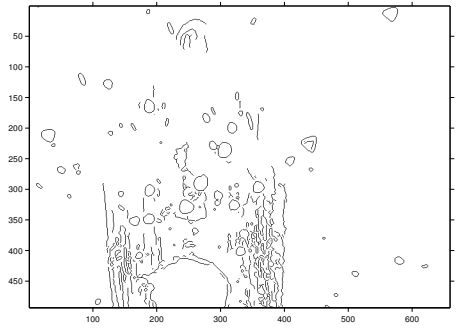

(b)

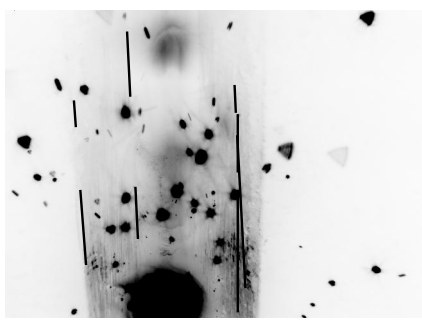

(c)

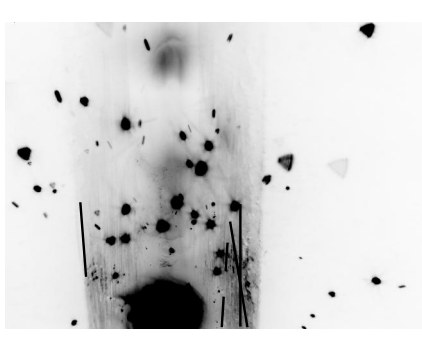

(d)

Fig. 2. Line Extraction using canny edge detector and Hough transform It can be seen that even with careful parameters selection automatically and manually, traditional edge detector + Hough transform technique still has difficulty to extract the edges of the seam. (a) Edge extracted by canny detector. The threshold was selected automatically. (b) Edge extracted by canny detector. The threshold was set to $0.1 \in[0,1]$. (c) Lines extracted by applying Hough transform to Fig.(a). (d) Lines extracted by applying Hough transform to Fig.(b). It should be noticed that the intensity of Fig.(c) and (d) was reversed to highlight the extracted lines. 
An energy functional $E(\mathbf{u}(s))$, parameterized by $\mathbf{u}(s)$, and including terms for internal, image dependent and external constraint forces is constructed such that the minimal energy is attained at the desired image feature, for instance, the boundary of an object. By minimizing its energy functional, the curve will converge to the desired image feature.

Kass et al. [5] proposed the first energy functional as the following:

$$
\begin{aligned}
E_{\text {snake }}= & \int_{0}^{1} \frac{1}{2}\left(\alpha(s)\left\|\mathbf{u}_{s}(s)\right\|^{2}+\beta(s)\left\|\mathbf{u}_{s s}(s)\right\|^{2}\right) \\
& +E_{\text {img }}(\mathbf{u}(s))+E_{\text {con }}(\mathbf{u}(s)) d s
\end{aligned}
$$

where $\mathbf{u}_{s}(s), \mathbf{u}_{s s}(s)$ denote the first and second derivative of the curve with respect to $s$, which forms the internal force. These two term are relative to the elasticity and curvature of the contour, respectively. This effect of each term can be controlled by parameters $\alpha(s), \beta(s)$.

$E_{\text {img }}(\mathbf{u}(s))$ represents image dependent force, for instance, $E_{i m g}(\mathbf{u}(s))$ is $-\left\|\nabla\left(G_{\sigma} * I\right)(\mathbf{u}(s))\right\|^{2}$, where $G_{\sigma}$ is a guassian with standard deviation $\sigma$ which is the kernel convolved with the input gray-level image $I$. By minimizing equation (1), the curve will be attracted to the maximum gradient region, i.e edges. The last term $E_{c o n}(\mathbf{u}(s))$ represents external constraint force which can offer users other options to control behaviors of snakes.

In [7], Williams and Shah introduced a greedy snake algorithm. Unlike the snake introduced by Kass et al. [5] solving the Euler-Lagrange equations derived from the energy function, greedy snake algorithm minimizes the energy function directly using greedy algorithm in a fully discrete manner. In each iteration, each controlled point of snake moves to new location which achieves the local minimum of the energy function in its neighborhood.

\section{B. A Line-Detected Snake for Seam Detection}

The proposed line-detected snake is represented as a parametric curve, illustrated in Fig. 3. The controlled points of snake are marked by nodes. Since we aim to extract the edges of the groove, i.e. four lines in the image, as shown in Fig. 1(c), a new energy function is proposed as following:

$$
E_{\text {snake }}^{*}=\int_{0}^{1}\left(\left\|\frac{d^{2} y}{d x^{2}}\right\|^{2}-\left\|\nabla\left(G_{\sigma} * I\right)(x(s), y(s))\right\|^{2}\right) d s
$$

In practice, snake performs in a discrete manner. The proposed energy function can be discretized and simplified as:

$$
\begin{gathered}
E_{\text {snake }}^{*} \approx \sum_{i=1}^{n-2}\left(\left|\frac{y_{i+1}-y_{i}}{x_{i+1}-x_{i}}-\frac{y_{i}-y_{i-1}}{x_{i}-x_{i-1}}\right|^{2}\right. \\
\left.-\left\|\nabla\left(G_{\sigma} * I\right)\left(x_{i}, y_{i}\right)\right\|^{2}\right)
\end{gathered}
$$

It should be noted that the first controlled point $\mathbf{u}_{0}$ and last one $\mathbf{u}_{n}$ will not be counted in when computing the energy function, because we cannot compute the second derivate of $y$ with respect to $x$ in these two points.

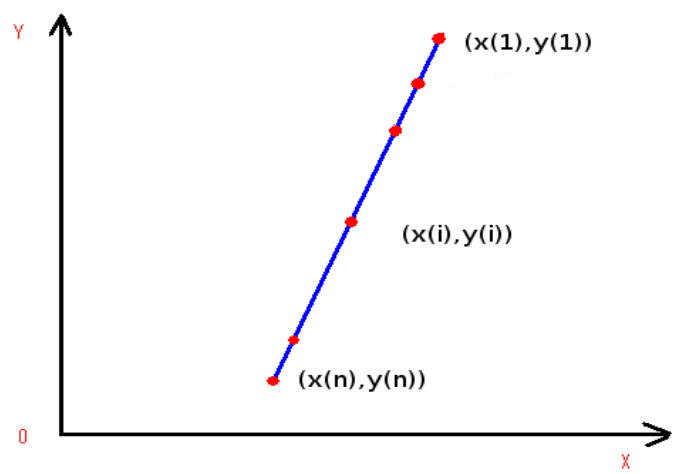

Fig. 3. Line-detected snake. This figure illustrates the discrete active contour for line detection. The controlled points of active contour are denoted by red nodes.

Equation (3) consists of two terms: the internal force term and the image dependent force term. To minimize these two terms, we follow two steps. First, we minimize the internal force term. Given a snake point $\mathbf{u}_{i}=\left(x_{i}, y_{i}\right)$ and its two neighbors $\mathbf{u}_{i-1}=\left(x_{i-1}, y_{i-1}\right), \mathbf{u}_{i+1}=\left(x_{i+1}, y_{i+1}\right)$, their relative positions can be categorized into two types, as shown in Fig. 4. In Fig. 4, $A, B, C$ are snake points, $B^{\prime}$ is the midpoint of $A$ and $C$. It is easy to see that one option to minimize the internal force term is to move the point $B$ towards $B^{\prime}$, i.e.

$$
\mathscr{U}_{i}[k]=\mathbf{u}_{i}[k]+\eta\left(\mathbf{u}_{i+1}[k]+\mathbf{u}_{i-1}[k]-2 \mathbf{u}_{i}[k]\right)
$$

where $k$ denotes the discrete step, $i$ is the index of the snake points, $\eta \in(0,1]$ is a scaling constant. As $\eta=0.5$, the point $B$ moves exactly to $B^{\prime}$. As $\eta<0.5$, the point $B$ moves towards $B^{\prime}$ but falls short of the point. As $\eta>0.5$, the point $B$ moves towards $B^{\prime}$ and crosses over the point.

Second, for minimization of the image dependent term, the gradient descent method was used [8]. The iterative equation can be derived as following:

$$
\mathbf{u}_{i}[k+1]=\mathscr{U}_{i}[k]-\xi \frac{\nabla\left(G_{\sigma} * I\right)\left(\mathbf{u}_{i}[k]\right)}{\left.\| \nabla\left(G_{\sigma} * I\right)\left(\mathbf{u}_{i}[k]\right)\right) \|}
$$

where $k$ denotes the discrete step, $i$ is the index of the snake points, $\xi$ is a scaling constant.

Combining equations (4) and (5), we can obtain a hybrid iterative equation to update the positions of the snake points in each iteration:

$$
\begin{aligned}
\mathbf{u}_{i}[k+1]=\mathbf{u}_{i}[k]+ & \eta\left(\mathbf{u}_{i+1}[k]+\mathbf{u}_{i-1}[k]-2 \mathbf{u}_{i}[k]\right) \\
& -\xi \frac{\nabla\left(G_{\sigma} * I\right)\left(\mathbf{u}_{i}[k]\right)}{\left.\| \nabla\left(G_{\sigma} * I\right)\left(\mathbf{u}_{i}[k]\right)\right) \|}
\end{aligned}
$$

The pseudocode of the proposed snake algorithm is showed as Procedure 1. The iteration of the algorithm can 


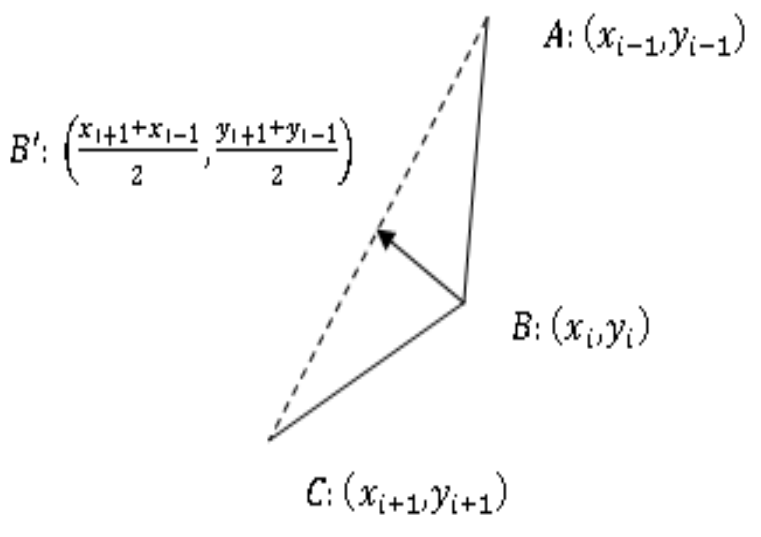

(a)

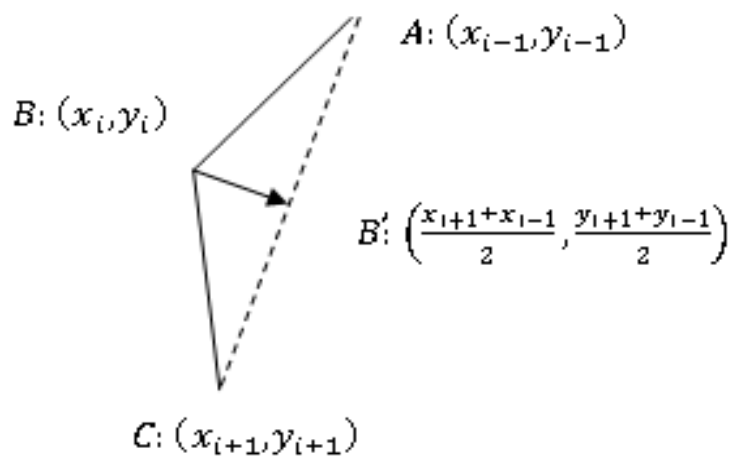

(b)

Fig. 4. The relative positions of one snake point and its two neighbors can be categoried as two types, as showed in (a) and (b). To maintain the line shape of snake, one option is to move $B$ towards $B^{\prime}$.

be stopped when either of the following stopping criteria is satisfied. 1)Predefined maximum of iterations is reached. 2)deviation of the control points from one iteration to the next is below one predefined small value.

\section{Image Denoising}

As we discussed above, the seam images without external illumination suffer from the interferences of high-intensity arc light and spatters. These interferences give rise to 'noises' that have some rather different characteristics from the the 'noise' which we deal with in traditional image processing case. According to some special characteristics of spatters in image, here we propose a new image-denosing technique to reduce the interferences of spatters during welding . Figure 6(a) and (b) show two typical successive frames retrieved from the image sequence. As we can see, the positions of spatters vary so much from frame to frame that none of them appears in the same position in two consecutive frames with a high probability. This provides us an opportunity to detect and remove the spatters in an efficient way.

The image sequence is considered as a multi-variable

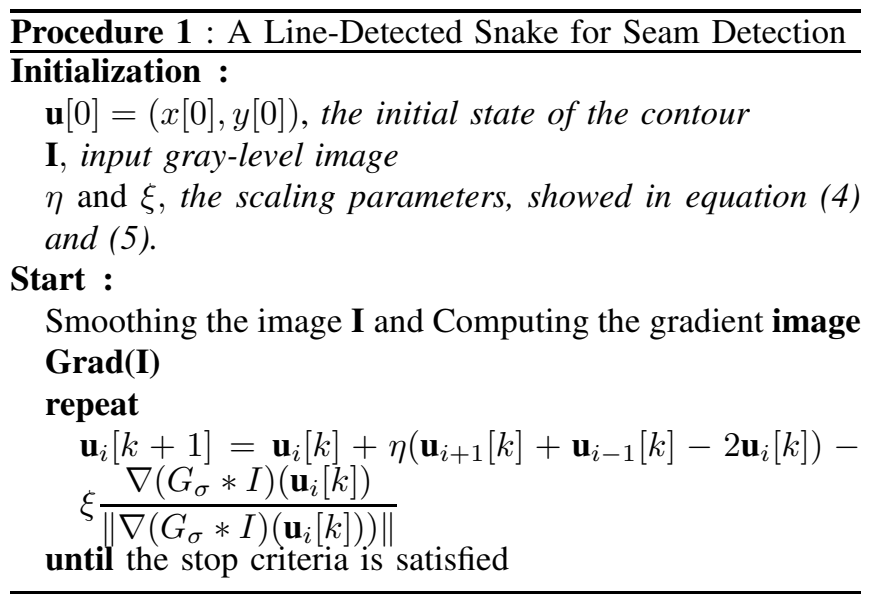

function $I(x, y, t)$, where $x$ and $y$ denote the position of a pixel in frame $t$. If a point $\left(x_{1}, y_{1}, t_{1}\right)$ belongs to a spatter, $\left.\frac{\partial I(x, y, t)}{\partial t}\right|_{\left(x_{1}, y_{1}, t_{1}\right)}$ will be very big with high probability. Therefore, with a careful threshold setting, we can locate the spatters by computing $\frac{\partial I(x, y, t)}{\partial t}$ for all pixes in each frame. The discretized equation is given in equation (7).

$\Delta I_{i}=\left\{\begin{aligned} I_{i}(x, y)-I_{i+1}(x, y) & \text { if } I_{i}(x, y)-I_{i+1}(x, y) \geq c \\ 0 & \text { if } I_{i}(x, y)-I_{i+1}(x, y)<c\end{aligned}\right.$

where $I_{i}(x, y)$ denotes the intensity of the frame $i . c$ is a threshold to mark our spatter area, i.e. if $I_{i}(x, y)-$ $I_{i+1}(x, y) \geq c$, then $(x, y)$ belongs to spatter area.

In order to remove the spatters, one option is to subtract $\Delta I_{i}$ from $I_{i}$ if the background of the image $I_{i}$ is similar to $I_{i+1}$. However, sometimes the intensities vary substantially from current frame to next frame. To further improve the quality of the denosied image, instead of using the frame right after the current frame, we pick the frame which has the most similar intensity to the current frame in its neighborhood.

$$
\Delta I_{i}=\left\{\begin{aligned}
I_{i}(x, y)-I_{s}(x, y) & \text { if } I_{i}(x, y)-I_{s}(x, y) \geq c \\
0 & \text { if } I_{i}(x, y)-I_{s}(x, y)<c
\end{aligned}\right.
$$

where $s=\arg \min \left|I_{t}-I_{i}\right|, t \in[i-l, i+l] . l$ is a integer deciding the size of the neighborhood. It should be noted that in the first few frames, we can just use the posterior neighbors on the time axis since it has very few preceding neighbors. Fig. 5(c) shows a denoising image of Fig.5(b). It can be seen that almost all spatters have been remove from the original image shown in Fig. 5(b). In addtion, because the denoising image may have a loss of contrast, histogram equalization is adopted to boost the contrast after denoising, as shown in Fig. 5(d).

\section{Proposed Image Analysis Scheme For Seam Tracking}

In this section, a prototype of the line-extraction scheme which can be used to process image sequences for seam tracking is proposed. Procedure 2 shows the psuedocode. 


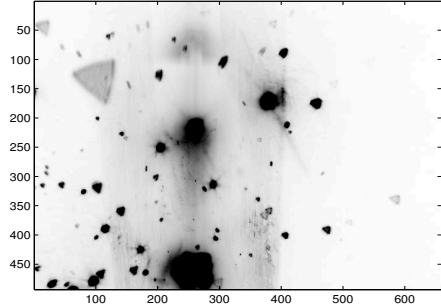

(a)

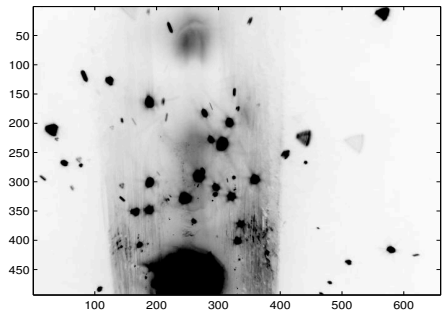

(b)

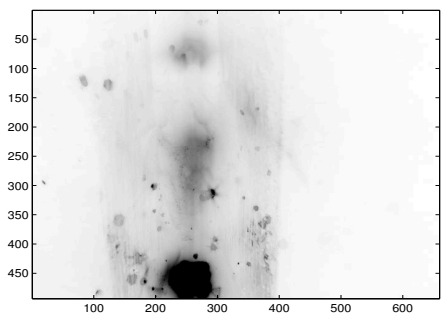

(c)

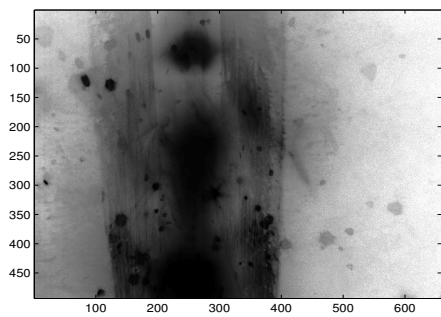

(d)

Fig. 5. (a) and (b) show two successive frames. (c) an denoising image obtained using the technique described in this section. (d) show the image after histogram equalization

The proposed snake technique can be considered as local search strategy. The initialization of the snake is very important. For the first few frames, the initial snake points can be located close to the edge of the frame, as shown in Fig. 7(b). And, an extra constraint force is used to drive the snake to the approximate position of the edge of the groove. For the latter frames, since the position of the edges of the groove vary little from frame to frame, we can use a more efficient way to initialize the snake: the final positions of the snake for current frame will be used as the initial positions of the snake for next frame after resampling, shown in equation (9).

$$
P_{\text {snake }}[k+1]=P_{\text {snake }}[k]+N(0, \sigma)
$$

where $P_{\text {snake }}[k]$ represents the positions of the snake for the $k$ th frame.

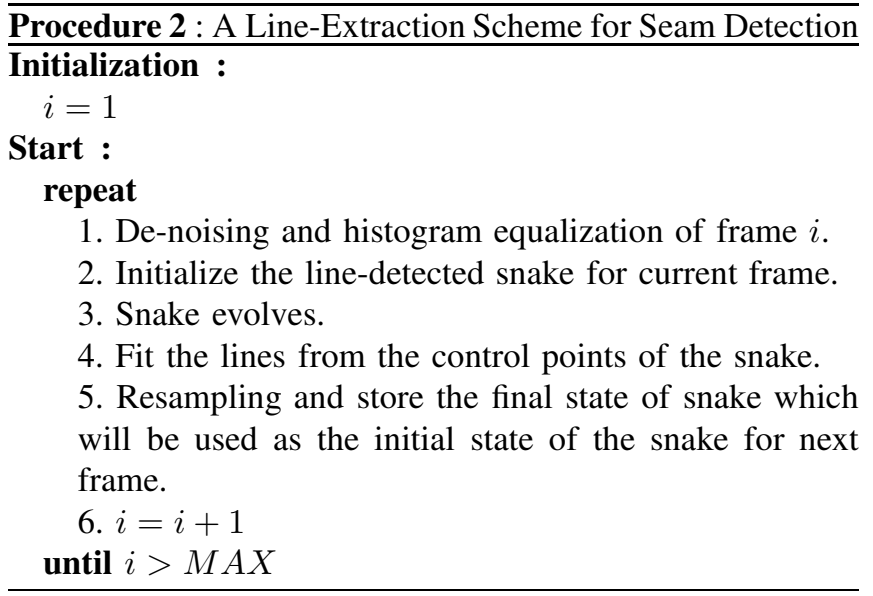

\section{EXPERIMENTS AND RESULTS}

\section{A. System Setup}

The experiment was conducted using a Posilica GC650E camera at an exposure time of $216 u s$. The welding process is the MAG welding using $82 \mathrm{Ar}-18 \mathrm{CO}_{2}$ as a shielding gas mixture. The wire feed rate is $2.5 \mathrm{~m} / \mathrm{min}$, the welding speed is $100 \mathrm{~mm} / \mathrm{min}$. The system setup is shown in Fig. 6 .

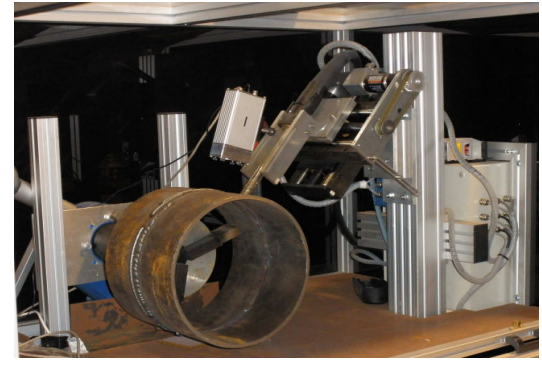

Fig. 6. System Setup

\section{B. Results and Analysis}

1) Test on a single image: To verify the feasibility of the proposed snake technique, several frames seriously corrupted by high-intensity spatters were retrieved from the image sequence during welding process. Fig. 7(a) shows one of the retrieved images. The parameters of the snake algorithm used in this experiment are in Table I.

The control points of snake were initialized as shown in Fig. 7(b). After 500 iterations, the snake converged to the edge of the groove, the straight line can be fitted from the final state of the snake, as shown in Fig. 7(c), (d), respectively.

By overlapping the fitted line with the original image and the edge image in Fig. 2(a), it can be seen that the edge of the groove was successfully extracted. Fig. 8 shows the overlapped images. 
TABLE I

PARAMETERS USED IN THIS EXPERIMENT

\begin{tabular}{cc}
\hline Parameters & Values \\
\hline$\eta$ & 0.1 \\
$\xi$ & 1 \\
$\sigma$ & 100 \\
$l$ & 10 \\
$c$ & 0 \\
\hline
\end{tabular}

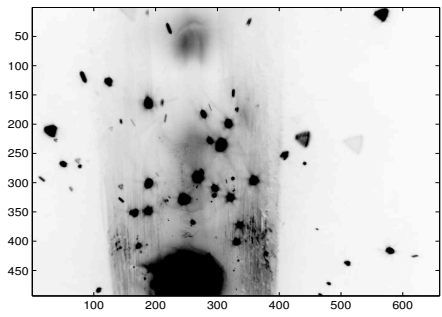

(a)

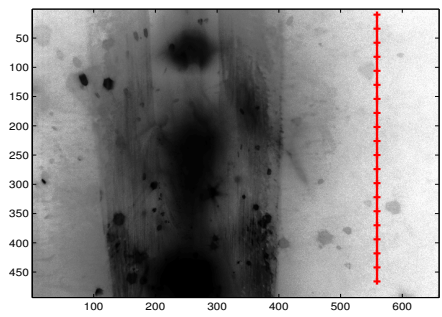

(b)

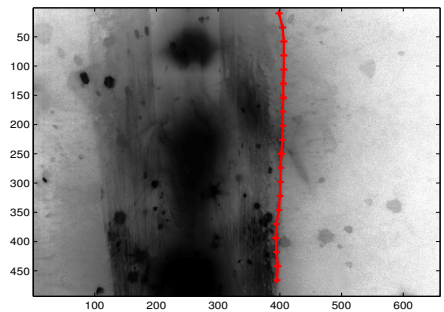

(c)

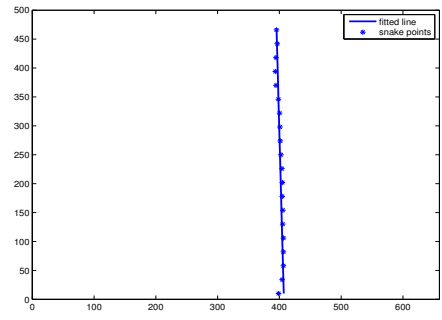

(d)

Fig. 7. (a) The original image. (b)The initial state. (c)The final state of snake. (d)The straight line was fitted from the control points of snake.

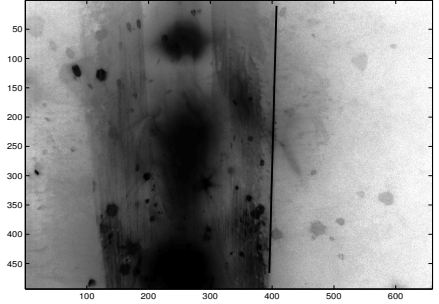

(a)

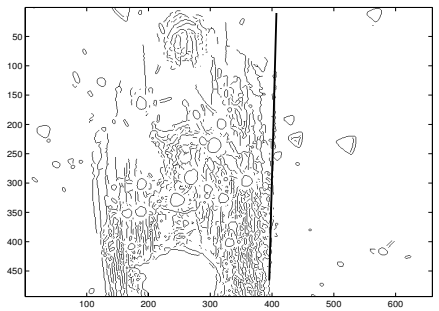

(b)

Fig. 8. The line extracted by snake. (a)Overlapped with the original image (b)Overlapped with the edge image showed in Figure 2.

2) Test on a image sequence: The offline algorithm is implemented in MATLAB, and tested on a video captured during welding. The results of processing five typical consecutive frames retrieved from the video are shown in Fig. 9. Without loss of generalization, we show the result of extracting the right edge of the groove. The initial states of the snake are shown in the second column of Fig. 9. It can be seen that after denoising and histgram equalization, the interference of high-intensity spatters has been reduced significantly. As we discussed above, the initialization of the snake for the first frame is different from the other four, as shown in Fig. 9 (b), (g), (l), (q), (v). This snake needed more time to converge to the edge after about 2000 iterations. For the other four, only about 100 iterations were used. From the last column of Fig. 9, we can see that the edge of the groove has been successfully extracted.

\section{CONClusions And Future Work}

Using laser scanner is a typical industrial solution for seam tracking which however still has some limitations. Some camera solutions without external illumination have also been presented. Due to the interferences of high-intensity arc light and spatters, the traditional edge detector + Hough transform technique has found great difficulty to extract the edges of the groove. In this paper, we proposed a new image analysis scheme based on active contour models for close-arc seam tracking, as well as a novel image denoising technique. The experimental results show the feasibility of this approach. For future work, a real-time version of this approach will be implemented. In addtion, based on extracted edges of the groove, we can calculate the geometrical features of the groove and then further embed these results as parameters into the control system. This approach will be 
fully investigated in real world application.

\section{REFERENCES}

[1] Peiquan, $\mathrm{Xu}$. "Application of circular laser vision sensor(CLVS) on welded seam tracking", Journal of Materials Processing Technology, p404-410, 2008.

[2] Haug, K, Pritschow,G. "Robust laser-stripe sensor for automated weldseam-tracking in the shipbuilding industry", Proc. of IECON'98,vol.2, p.1236-1241,1998.

[3] D. Xu, Z, Jiang, L, Wang, M, Tan."Features extraction for structured light image of welding seam with arc and splash disturbance". Proc. of ICARCV, vol.3, p1559-1563,2004.

[4] Duda, R.O. and Hart, P.E. "Use of the Hough Transform to Detect Lines and Curves in Pictures", Comm. ACM, Vol 15, No. 1, pp. 1115, 1972.

[5] Michael Kass, Andrew Witkin and Demetri Terzopoulos, "Snakes: Active Contour Models", International Journal of Computer Vision, p321-331, (1988).

[6] J. Canny. "A computational approach to edge detection", IEEE Trans. on Pattern Analysis and Machine Intelligence. Vol. 8, pp 679-698, 1986.

[7] D. J. Williams and M. Shah. "A fast algorithm for active contours and curvature estimation". CVGIP: Image Understanding, Vol. 55, pp. 1426, 1992.

[8] Mordecai Avriel. "Nonlinear Programming: Analysis and Methods". Dover Publishing. ISBN 0-486-43227-0. 2003. 


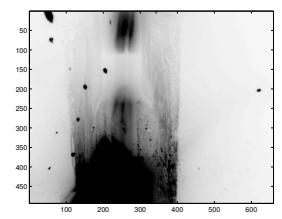

(a)

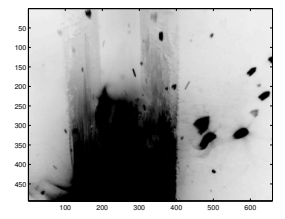

(f)

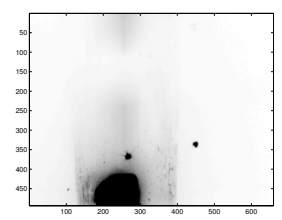

(k)

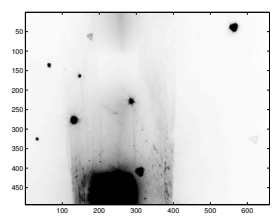

(p)

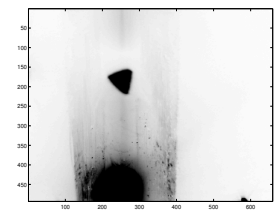

(u)

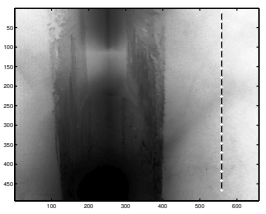

(b)

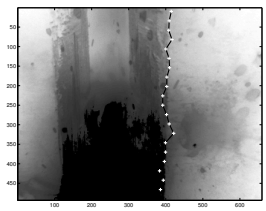

(g)

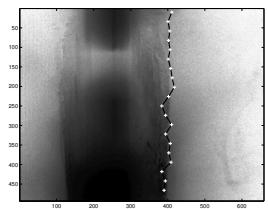

(1)

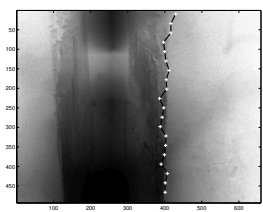

(q)

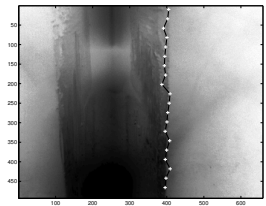

(v)

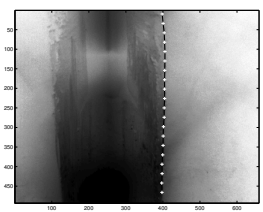

(c)

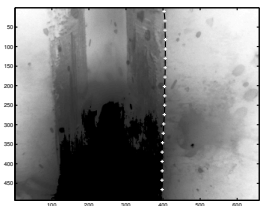

(h)

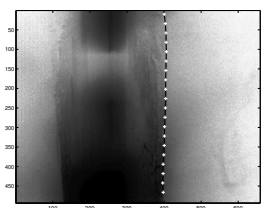

(m)

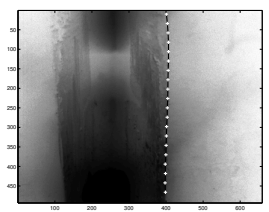

(r)

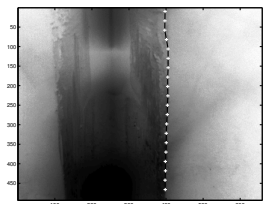

(w)

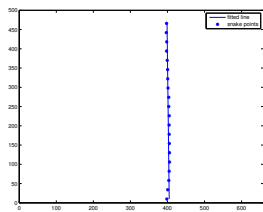

(d)

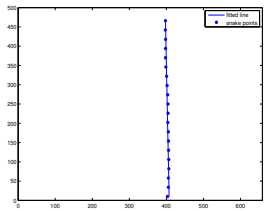

(i)

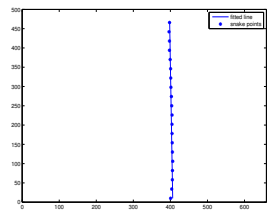

(n)

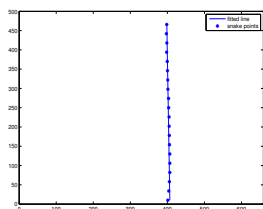

(s)

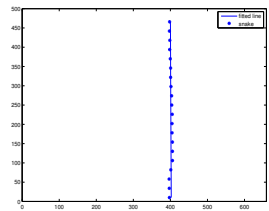

(x)

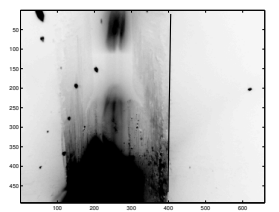

(e)

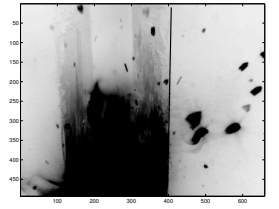

(j)

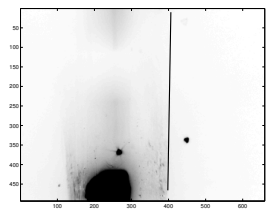

(o)

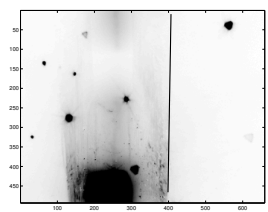

(t)

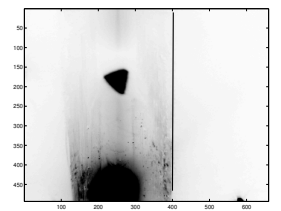

(y)

Fig. 9. Test the proposed scheme in a image sequence. Five consecutive frames were retrieved from the image sequence, as shown in the first column. The initial states of the snakes for each frame are shown in second column. The third and fourth columns show the final state of the snakes and the fitted lines, respectively. The last column shows the extracted lines overlapped with the original images. 